TecnoHumanismo. Revista Científica

Setiembre - Noviembre 2021

Volumen 1 / No.3

ISSN: $2710-2394$

pp. $65-74$

https://doi.org/10.53673/th.v2i2.102

https://tecnohumanismo.online

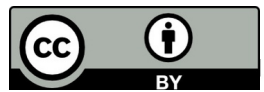

\title{
Calidad percibida y satisfacción de los egresados de la Facultad de Ciencias de una Universidad Nacional
}

Perceived quality and satisfaction of graduates of the Faculty of Sciences of a National University

Qualidade percebida e satisfação dos graduados da Faculdade de Ciências de uma Universidade Nacional

\section{ARTÍCULO GENERAL}

Dr. Jorge Antonio Acosta Piscoya

jacosta@unprg.edu.pe

https://orcid.org/0000-0002-2280-2974

Universidad Nacional Pedro Ruiz Gallo

Lambayeque-Perú
Msc. Débora Esther Mejía Pacheco dmejia@unprg.edu.pe

https://orcid.org/0000-0003-0543-1021

Universidad Nacional Pedro Ruiz Gallo

Lambayeque-Perú

Recibido 07 de Abril 2021 | Arbitrado y aceptado 19 de Junio 2021 | Publicado en 03 Setiembre 2021

\section{RESUMEN}

El Objetivo de la presente investigación fue determinar la calidad y satisfacción de los egresados de la Facultad de Ciencias Físicas y Matemáticas (FACFyM) de la Universidad Nacional Pedro Ruiz Gallo (UNPRG) de Lambayeque, donde participaron 113 egresados de las cinco escuelas profesionales que conforman la FACFyM (Estadística, Matemáticas, Física, Ingeniería en computación e Informática, Ingeniería electrónica), el 76,1\% de los participantes son hombres, los ítems más valorados corresponden a la dimensión calidad percibida de la sub dimensión responsabilidad y seguridad, y en la dimensión satisfacción lo ítems más valoran están relacionados con la sub dimensión proceso académico y la Docencia. El instrumento utilizado fue la hoja de actualización de datos del graduado/titulado de la oficina de seguimiento del egresado, el cual nos dio una alfa de Cronbach de 0.946 y un KMO 0.886, así mismo al realizar el análisis factorial se determinó que la calidad y satisfacción de los egresados de la FACFyM se puede explicar en cinco factores con una varianza explicada del $69,437 \%$, se determinó que el más representativo es el factor uno que agrupa todos los ítems correspondientes a la Calidad percibida por el egresado donde destacan la formación recibida por parte del docente.

Palabra Clave: Calidad Percibida y Satisfacción de egresados.

\section{ABSTRACT}

The objective of this research was to determine the quality and satisfaction of the graduates of the Faculty of Physical and Mathematical Sciences (FACFyM) of the Pedro Ruiz Gallo National University (UNPRG) of Lambayeque, where 113 graduates of the five professional schools that make up the FACFyM (Statistics, Mathematics, Physics, Computer and Informatics Engineering, Electronic Engineering), $76.1 \%$ of the participants are men, the most valued items correspond to the perceived quality dimension of the responsibility and safety sub-dimension, and in In the satisfaction dimension, the items they value the most are related to the academic process and Teaching subdimension. The instrument used was the data update sheet of the graduate / graduate from the graduate follow-up office, which gave us a Cronbach's alpha of 0.946 and a KMO 0.886 , likewise when performing the factor analysis it was determined that the quality and Satisfaction of the graduates of the FACFyM can be explained in five factors with an explained variance of $69.437 \%$, it was determined that the most representative is factor one that groups all the items corresponding to the Quality perceived by the graduate where the training received by the graduate stands out. part of the teacher. Keyword: Perceived Quality and Satisfaction of graduates.

\section{RESUMO}

O objetivo desta pesquisa foi determinar a qualidade e satisfação dos graduados da Faculdade de Ciências Físicas e Matemáticas (FACFyM) da Universidade Nacional Pedro Ruiz Gallo (UNPRG) de Lambayeque, onde 113 graduados das cinco escolas profissionais que compõem no FACFyM (Estatística, Matemática, Física, Engenharia Informática e Informática, Engenharia Eletrônica), 76,1\% dos participantes são homens, os itens mais valorizados correspondem à dimensão qualidade percebida da subdimensão responsabilidade e segurança, e na satisfação dimensão, os itens que mais valorizam estão relacionados ao processo acadêmico e subdimensão Ensino. O instrumento utilizado foi a ficha de atualização de dados do graduado / graduado do escritório de acompanhamento de pós-graduação, que nos deu um alfa de Cronbach de 0,946 e um KMO de 0,886, da mesma forma ao realizar a análise fatorial foi determinado que a qualidade e satisfação dos os graduados da FACFyM podem ser explicados em cinco fatores com uma variância explicada de 69,437\%, determinou-se que o mais representativo é o fator aquele que agrupa todos os itens correspondentes à Qualidade percebida pelo graduado onde se destaca a formação recebida pelo graduado .parte do professor. Palavra chave: Qualidade percebida e Satisfação dos egressos. 


\section{Introducción}

La evaluación continua y sistemática de la institución universitaria es una actividad esencial para innovar, descubrir nuevos métodos de enseñanza - aprendizaje y optimizar recursos, a lo cual contribuyen los estudios de seguimiento a egresados. Estos estudios permiten conocer información acerca del desempeño profesional, opiniones y sugerencias acerca de la calidad de la educación recibida y de las nuevas demandas del mercado laboral y del medio social (CNA, 1988; De la Cruz, Macedo y Torres, 1996; Maritza Ferrera s.f. citada por Landazabal, Melo y Meses, 2000; Mendoza, 2003). Así, los estudios de seguimiento e impacto de egresados se convierten en un mecanismo para establecer una relación de doble vía entre la institución y los egresados y benefician a todas las partes involucradas, desde las instituciones hasta la comunidad, incluyendo a los egresados y a los profesionales en formación, por cuanto su propósito es contribuir a mejorar la calidad de la educación, a fin de aportar a la solución de problemas. citado por Aldana et al (2008).

En ese contexto con la promulgación de la Ley No 30220, Ley Universitaria, el Ministerio de Educación (MINEDU) asume la rectoría de la Política de Aseguramiento de la Calidad de la Educación Superior Universitaria. Además, se crea la Superintendencia Nacional de Educación Superior Universitaria (SUNEDU), y se introduce el licenciamiento obligatorio y renovable de las universidades. Bajo este marco con Resolución Nº177-2019-D/FACFYM se implementa la Oficina Administrativa de seguimiento del egresado de la FACFyM, la cual tiene como función principal la recopilación de información sobre el desarrollo profesional, personal y social de los señores egresados de las cinco escuelas profesionales (Estadística, Matemáticas, Física, Computación e Informática e Ingeniería Electrónica) que conforman la Facultad de Ciencias Físicas y Matemáticas, en la actualidad no existe un ente que se encargue de la evaluación de la inserción laboral del egresado de nuestra facultad, por lo que existe la necesidad de contar con información básica y fundamental para la programación y planificación del desarrollo de la institución acorde con la realidad, es conveniente e indispensable realizar un diagnóstico de la calidad percibida y satisfacción de los egresado de la Facultad de Ciencias Físicas y Matemáticas de la UNPRG. 


\section{Antecedentes}

Pereira, M. (2014). En su tesis doctoral concluye que la evaluación de la calidad de las universidades ha llegado a cobrar un gran protagonismo debido a la importancia que hoy día se le concede a la educación superior como herramienta fundamental para la consecución del crecimiento económico y el bienestar social. Una importancia que se espera vaya en aumento a medida que los países de nuestro entorno socio-económico implanten progresivamente sistemas productivos fundamentados en las premisas de la economía del conocimiento, pues 215 estos sistemas requieren para su éxito de grandes cantidades de personas altamente calificadas y motivadas para seguir formándose a lo largo de toda la vida.

Gómez, S. et al. (2019). En su artículo científico Modelo de satisfacción del egresado universitario, entre sus principales conclusiones del modelo propuesto logró demostrar convergencia entre sus respectivas variables, la carga factorial estandarizada fue superior a 0,7 ; por otra parte, el promedio de las cargas de los indicadores sobre cada factor fue superior a 0,7 para todos los constructos, lo que indica que con la modelación se pudieron determinar constructos de satisfacción como «imagen que proyecta hacia el entorno» o «pertinencia del currículo», que determinan el grado de satisfacción que expresa el egresado ante la sociedad y la institución misma.

\section{Metodos y materiales}

La presente investigación es censal por lo que la población y muestra son iguales se analizaron todas las encuestas presenciales y virtuales llenadas por los egresados de la Facultad de Ciencias Físicas y Matemáticas que llegaron a realizado su trámite en la oficina de grados y títulos durante los años 2019 - 2020. Se usaron fuente primaria. la técnica de observación y encuesta, con la cal se creó una base de datos, usando hoja de cálculo de Excel con apoyo de un software estadístico, primero se realizó un análisis diagnostico utilizando la Estadística descriptiva e inferencial, así mismo el análisis factorial exploratorio con rotación varimax. 


\section{Resultados}

Dentro de los resultados encontrados al realizar el análisis exploratorio de los egresados que participan en el estudio de la calidad percibida y satisfacción del estudiante, tenemos:

\section{Tabla 1.}

Egresados por sexo según escuela profesional

\begin{tabular}{|c|c|c|c|c|c|c|}
\hline \multirow[b]{3}{*}{ Escuela Profesional } & \multicolumn{4}{|c|}{ SEXO } & \multicolumn{2}{|c|}{ Total } \\
\hline & \multicolumn{2}{|c|}{ HOMBRE } & \multicolumn{2}{|c|}{ MUJER } & & \\
\hline & $\mathrm{N}^{\circ}$ & $\%$ & $\mathrm{~N}^{\circ}$ & $\%$ & $\mathrm{~N}^{\circ}$ & $\%$ \\
\hline ESTADISTICA & 2 & 1,8 & 11 & 9,7 & 13 & 11,5 \\
\hline FISICA & 14 & 12,4 & 2 & 1,8 & |16 & 14,2 \\
\hline $\begin{array}{l}\text { Ing. } \\
\text { COMPUTACIÓN }\end{array}$ & 23 & 20,4 & 5 & 4,4 & 28 & 24,8 \\
\hline $\begin{array}{l}\text { Ing. } \\
\text { ELECTRÓNICA }\end{array}$ & 34 & 30,1 & 1 & 0,9 & 35 & 31,0 \\
\hline MATEMATICAS & 13 & 11,5 & 8 & 7,1 & 21 & 18,6 \\
\hline Total & 86 & 76,1 & 27 & 23,9 & 113 & 100,0 \\
\hline
\end{tabular}

Nota: Base de encuesta seguimiento del egresado

Los resultados que se muestran en la tabla 1, en el estudio participan 113 egresados de las diferentes escuelas el $76,1 \%$ son hombres, el $31 \%$ pertenecen a la escuela profesional de ingeniería electrónica, el $24,8 \%$ a la escuela de ingeniería de computación e informática, el 18,6\% a la escuela de Matemáticas, 14,2\% a la escuela profesional de Física y el 11,5\% a la escuela profesional de Estadística

Tabla 2.

Análisis descriptivo de la satisfacción y calidad percibida por el egresado

\begin{tabular}{lllll}
\hline \hline & Media & D.E & C.V & N \\
\hline Satisfacción & 47.4690 & 8.65104 & 0.18 & 113 \\
Calidad & 53.4956 & 12.82306 & 0.24 & 113 \\
\hline \hline
\end{tabular}

Nota: Base de encuesta seguimiento del egresado 
Los ítems correspondientes a la calidad percibida fueron más valorados que la satisfacción (53.4956 > 47.4690), así mismo las respuestas de los ítems de calidad presentan una mayor dispersión que las de satisfacción $(0.24>0.18)$.

Tabla 3.

Ítems de satisfacción y calidad más valorado.

\begin{tabular}{lcccc}
\hline \hline & $\mathrm{N}$ & Media & D.E & C.V \\
\hline $\begin{array}{l}\text { S.3. Satisfacción con las asesorías de } \\
\text { tesis recibida. }\end{array}$ & 113 & 4.01 & 0.901 & 0.22 \\
$\begin{array}{l}\text { S.4. Satisfacción con el trámite para } \\
\text { obtener el grado de bachiller, titulo. }\end{array}$ & 113 & 3.94 & 1.112 & 0.28 \\
$\begin{array}{l}\text { C.7. Los profesores de la UNPRG } \\
\text { están dispuestos siempre para ayudar a }\end{array}$ & 113 & 3.79 & 1.097 & 0.29 \\
$\begin{array}{l}\text { los estudiantes } \\
\begin{array}{l}\text { C.8. El comportamiento de los } \\
\text { profesores de la UNPRG le inspira } \\
\text { confianza }\end{array}\end{array}$ & 113 & 3.72 & 1.048 & 0.28 \\
\hline
\end{tabular}

Nota: Base de encuesta seguimiento del egresado

Entre los ítems más valorados por los egresados de la cinco escuelas profesionales de la FACFyM, están el ítem de satisfacción relacionado con la asesoría de tesis recibida por los docentes, y con el tramite para obtener el grado de Bachiller y titulo profesional; así mismo en los ítems relacionados a calidad el mas valoran a la disposición del docente de la UNPRG para ayudarlos y la confianza que le inspira.

TABLA 4.

Análisis factorial de la varianza explicada $i$

\begin{tabular}{|c|c|c|c|c|c|c|c|c|}
\hline \multirow{3}{*}{$\begin{array}{l}\text { Component } \\
\mathrm{e}\end{array}$} & \multicolumn{3}{|c|}{ Autovalores iniciales } & \multicolumn{3}{|c|}{\begin{tabular}{|l|} 
Sumas de cargas al \\
cuadrado de la extracción
\end{tabular}} & \multicolumn{2}{|c|}{$\begin{array}{l}\text { Sumas de cargas al } \\
\text { cuadrado de la rotación }\end{array}$} \\
\hline & & $\begin{array}{l}\% \text { de } \\
\text { varianz }\end{array}$ & $\begin{array}{l}\% \\
\text { acumulad }\end{array}$ & & $\begin{array}{l}\% \text { de } \\
\text { varianz }\end{array}$ & $\begin{array}{l}\% \\
\text { acumulad }\end{array}$ & $\begin{array}{l}\% \text { de } \\
\text { varianz }\end{array}$ & $\begin{array}{l}\% \\
\text { acumulad }\end{array}$ \\
\hline & Total & $\mathrm{a}$ & o & Total & $\mathrm{a}$ & o & Totala & o \\
\hline 1 & $\begin{array}{l}11.71 \\
3\end{array}$ & 41.834 & 41.834 & $\begin{array}{l}11.71 \\
3\end{array}$ & 41.834 & 41.834 & \begin{tabular}{|ll}
9.25 & 33.062 \\
7 &
\end{tabular} & 33.062 \\
\hline 2 & 3.946 & 14.093 & 55.927 & 3.946 & 14.093 & 55.927 & $\begin{array}{ll}4.28 & 15.299 \\
4 & \end{array}$ & 48.361 \\
\hline 3 & 1.719 & 6.140 & 62.067 & 1.719 & 6.140 & 62.067 & $\begin{array}{ll}2.91 & 10.423 \\
9 & \end{array}$ & 58.784 \\
\hline
\end{tabular}


Jorge Antonio Acosta Piscoya, Débora Esther Mejía Pacheco

\begin{tabular}{|c|c|c|c|c|c|c|c|}
\hline 4 & 1.062 & 3.792 & 65.859 & |1.062 3.792 & 65.859 & $\begin{array}{ll}1.63 & 5.835 \\
4\end{array}$ & 64.619 \\
\hline 5 & 1.002 & 3.578 & 69.437 & 1.0023 .578 & 69.437 & $\begin{array}{ll}1.34 & 4.818 \\
9 & \end{array}$ & 69.437 \\
\hline 6 & .894 & 3.195 & 72.632 & & & & \\
\hline 7 & .820 & 2.929 & 75.561 & & & & \\
\hline 8 & .747 & 2.667 & 78.227 & & & & \\
\hline$\ldots$ & $\ldots$ & $\ldots$ & ...... & & & & \\
\hline 28 & .077 & .273 & 100.000 & & & & \\
\hline
\end{tabular}

Método de extracción: análisis de componentes principales.

Los resultados obtenidos nos permiten determinar que si es posible realizar el análisis factorial, obteniendo una fiabilidad del instrumento de 0.946 y un KMO de 0.889, y que se puede agrupar en seis componentes o factores (grafico de sedimentación), con una varianza explicada del 69,437\%.

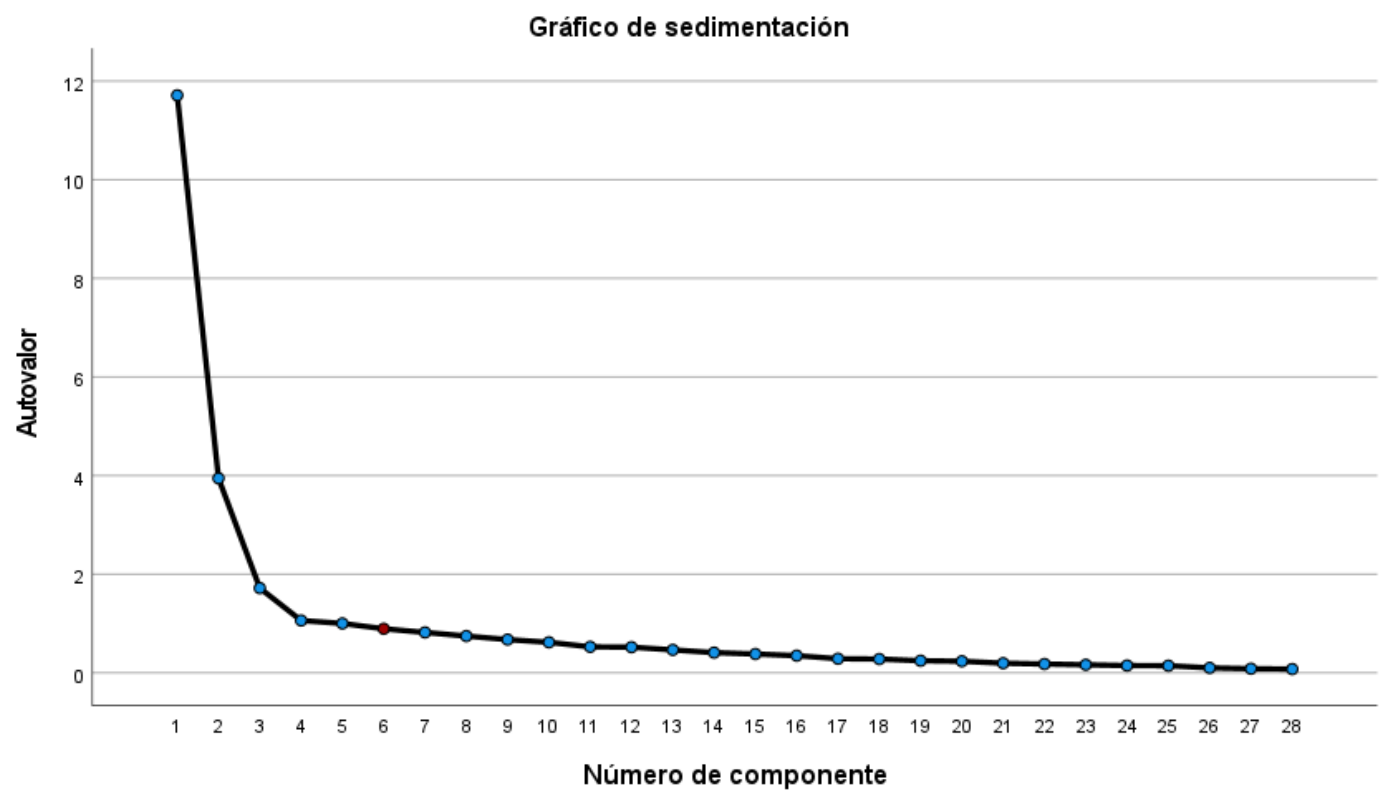


Tabla 5.

Matriz de componentes rotados

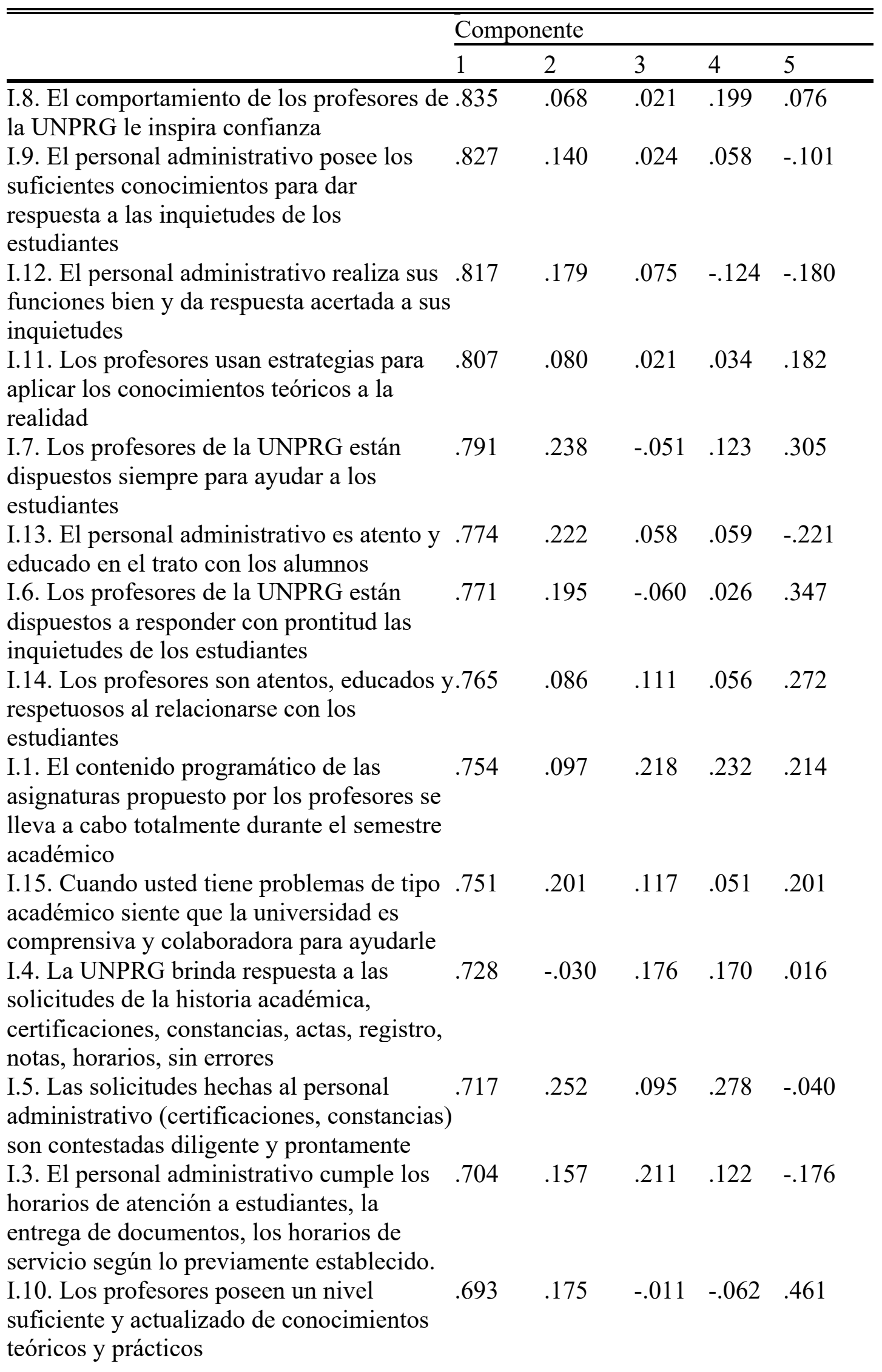


$\begin{array}{llllll}\text { I.2. Los servicios complementarios de la } & .676 & .038 & .226 & .026 & -.030\end{array}$ educación (biblioteca, médicoodontológico, cafetería, secretaria) y horarios se prestan según lo promedio.

I.16. Satisfacción respecto al proceso de matrícula cada ciclo que ha cursado I.27. Satisfacción con la atención por parte .190 de su director de escuela I.28. Satisfacción con la atención por parte .410 de la secretaria de su facultad Escuela I.22. Satisfacción con el trato por parte de .22 los docentes en clase las fechas de entrega de notas, las actividades extracurriculares son cumplidos por los profesores en el tiempo que se ha programado

I.21. Satisfacción con el nivel profesion de los docentes

I.26. Satisfacción con el servicio y material .116 bibliográfico de la biblioteca

I.24. Satisfacción con las distribuciones y $\quad .160 \quad .224$ equipamiento de los laboratorios

I.23. Satisfacción con el aula, mobiliario y $\quad .222$ equipos audiovisuales.

I.25. Satisfacción con las instalaciones $\quad-.036$ deportivas

I.18. Satisfacción con las asesorías de tesis .216 recibida.

I.19. Satisfacción con el trámite para $.104 \quad .496$ $.199 \quad .050 \quad .106$ obtener el grado de bachiller, licenciado, Magister, Doctor

$\begin{array}{llllll}\text { I.20. Las asignaturas de su plan curricular } & .260 & .315 & .386 & .300 & .393\end{array}$
fueron suficientes para su formación.

El método de rotación factorial permitió determinar que el factor uno es el más representativo que agrupa a todos los ítems correspondiente a la calidad percibidas, y el factor II al V agrupa a los ítems correspondiente a Satisfacción de los egresados de la FACFyM. 


\section{Discusion}

- Los resultados encontrados en la presente investigación son similares a los encontrados por otros investigadores como la de Pereira (2014), Alves y Reposo (2004), donde concluyen que la supervivencia de las instituciones de enseñanza superior depende de la satisfacción de sus egresados. Esto se refleja en nuestra investigación debido a que el factor de mayor representación es la calidad percibida por el egresado, a mayor calidad mayor satisfacción.

- Si bien las cargas factoriales en la presente investigación son inferiores a las encontradas por Gómez (2019), el primer factor que es el que mejor explica la satisfacción de egresado tiene cargas superiores a 0.6 , donde la mayoría de los ítems muestran su satisfacción con la enseñanza recibida por parte de sus docentes.

\section{Conclusiones}

La calidad percibida por los egresados de la escuela de Estadística, Matemáticas, Física, Ingeniería en computación e informática, e Ingeniería en electrónica, se basa en el trato y comportamiento que tiene el docente.

La Satisfacción percibida por los egresados de la escuela de Estadística, Matemáticas, Física, Ingeniería en computación e informática, e Ingeniería en electrónica, se basa en la asesoría de tesis y con el trámite para obtener el grado de bachiller o el título.

$>$ La calidad y satisfacción percibida por el egresado de la FACFyM se puede explicar en cinco factores con una varianza explicada del 69,437\%.

De los cinco factores encontrados es el factor uno el que mejor explica la calidad percibida por el egresado que agrupa los 15 ítems considerados en la dimensión calidad.

\section{Bibliogafia}

Aldana, G., Morales, F., Aldana, J., Sabogal, F., y Ospina, A. (2008). Seguimiento a egresados, su importancia para las instituciones de educación superior. https://dialnet.unirioja.es/servlet/articulo?codigo $=3701001$ 
Ahumada, F. \& Obregón, C. (2017). Seguimiento a Egresado: Un estudio diagnostico en la Benemerita y Centenaria Escuela Normal del Estado de San Luis Potosí.

\section{http://www.comie.org.mx/congreso/memoriaelectronica/v14/doc/1227.pdf}

Cabos y D’Angelo (2019). “Satisfacción del Estudiante Egresado En El Programa De Pregrado Adulto Trabajador De Una Universidad Del Norte Del Perú, 2018”. https://repositorio.upn.edu.pe/bitstream/handle/11537/23654/Cabos\%20Villa\%2C \%20Luigi $\% 20$ Vatslav\%20\%20D $\%$ C2\%B4Angelo\%20Panizo $\% 2 \mathrm{C} \% 20 \mathrm{Mar} \% \mathrm{C} 3 \% \mathrm{ADa} \% 20 \mathrm{del} \% 20 \mathrm{Carmen}$. pdf? sequence $=1 \&$ isAllowed $=\mathrm{y}$

García, C., Treviño, A. \& Banda, F. (2018). Caracterización del seguimiento de egresados universitarios.

http://scielo.sld.cu/pdf/reds/v7n1/2308-0132-reds-7-01-23.pdf

Gómez, S., Palacios, L., Berrio, J., Gaviria, S, Quinceno, L., y Figueroa, P. (2019).

Modelo de satisfacción de egresados universitarios: Un estudio de caso. https://revistas.itm.edu.co/index.php/revista-cea/article/view/1443/1337

Pereira, M (2014). Educación superior universitaria: calidad percibida y satisfacción de los egresados. Tesis doctoral Universidad Da Coruña. https://ruc.udc.es/dspace/bitstream/handle/2183/12349/PereiraPuga_Manuel_TD 2014.pdf? sequence $=2 \&$ isAllowed $=y$

Sánchez, Q. (2018). Satisfacción estudiantil en educación superior. Validez de su medición. https://repository.usergioarboleda.edu.co/bitstream/handle/11232/1027/SATISFA $\mathrm{CCI} \% \mathrm{C3} \% 93 \mathrm{~N} \% 20 \mathrm{ESTUDIANTIL} . p d f ?$ sequence $=4 \&$ isAllowed $=\mathrm{y}$

Seañez, J. (2010). Estudio del seguimiento del egresado 2006 -2010.

http://diex.uach.mx/seguimiento_de_egresados/Estudio $\% 20 \mathrm{de} \% 20$ Seguimiento $\% 20 \mathrm{de} \%$ 20Egresados $\% 202006-2010 \% 20 \mathrm{UACH} \% 20 \% 20$ Institucional $\% 20$ consulta.pdf 